\title{
Región y patrimonio biocultural de los ch'oles de Tacotalpa, Tabasco
}

\section{Region and biocultural heritage of the ch'oles of Tacotalpa, Tabasco}

\author{
MORALES-VALENZUELA, Guadalupe $\dagger^{*}$, VILLEGAS-RAMÍREZ, María Isabel, CARRILLO- \\ CONTRERAS, Jaime y JIMÉNEZ-ARANO, Carlos Martín
}

Universidad Intercultural del Estado de Tabasco. Carretera Oxolotán-Tacotalpa, Tacotalpa, Tabasco. CP. 86890.

ID $1^{\mathrm{er}}$ Autor: Guadalupe, Morales-Valenzuela / ORC ID: 0000-0003-4289-5415, CVU CONACYT ID: 37866

ID $1^{\mathrm{er}}$ Coautor: María Isabel, Villegas-Ramírez / ORC ID: 0000-0001-8912-5261, CVU CONACYT ID: 867564

ID $2^{\text {do }}$ Coautor: Jaime, Carrillo-Contreras / ORC ID: 0000-0001-6343-8761

ID $3^{\text {er }}$ Coautor: Carlos Martín, Jiménez-Arano / ORC ID: 0000-0002-3971-7979

DOI: $10.35429 / J O C S .2019 .19 .6 .20 .30$

Recibido: 03 de Mayo, 2019; Aceptado 30 de Junio, 2019

\begin{abstract}
Resumen
El objetivo de este trabajo fue delimitar y caracterizar la región que ocupan los Ch'oles de Tacotalpa, Tabasco, así como identificar su patrimonio biocultural. Para delimitar la región se consideró el criterio de localidades con más del $40 \%$ de hablantes de la lengua $\mathrm{CH}^{\prime}$ ol y los núcleos ejidales ocupados por dichas localidades. La caracterización de la región se realizó mediante revisión de fuentes secundarias y entrevistas, y la identificación del patrimonio biocultural mediante entrevistas y observación. La región la integran 31 localidades en 22 núcleos ejidales con una superficie de 22 414. 791 hectáreas. El $79.43 \%$ de los habitantes de las localidades que integran esta región biocultural hablan la lengua CH'ol y mantienen costumbres y tradiciones relacionadas al catolicismo y a prácticas ancestrales. La situación ambiental en esta región está relacionada con los recursos naturales y la cultura. La economía de esta región está centrada en las actividades agropecuarias, básicamente en la producción de la milpa y un crecimiento de la ganadería bovina y monocultivos (palma de aceite y teca). El patrimonio biocultural de la región está representado por la agricultura tradicional, la medicina tradicional, aprovechamiento de los recursos naturales y danzas y rituales.
\end{abstract}

Territorio, Patrimonio, Biocultural

\begin{abstract}
The objective of this work was to define and characterize the region occupied by the Ch'oles de Tacotalpa, Tabasco, as well as identify their biocultural heritage. To delimit the region, the criterion of localities with more than $40 \%$ of speakers of the CH'ol language and the ejido nuclei occupied by these localities was considered. The characterization of the region was carried out by review of secondary sources and interview, and identification of the biocultural heritage through interviews and observation. The region is made up of 31 localities in 22 ejido cores with an area of $22,414,791$ hectares. $79.43 \%$ of the inhabitants of the towns that make up this biocultural region speak the $\mathrm{CH}$ 'ol language and maintain customs and traditions related to Catholicism and ancestral practices. The environmental situation in this region is related to natural resources and culture. The economy of this region is focused on agricultural activities, basically in the production of the cornfield and the growth of cattle and monocultures (palm oil and teak). The biocultural heritage of the region is represented by traditional agriculture, traditional medicine, use of natural resources and dances and rituals.
\end{abstract}

Territory, Heritage, Biocultural

Citación: MORALES-VALENZUELA, Guadalupe, VILLEGAS-RAMÍREZ, María Isabel, CARRILLO-CONTRERAS, Jaime y JIMÉNEZ-ARANO, Carlos Martín. Región y patrimonio biocultural de los ch'oles de Tacotalpa, Tabasco. Revista de Sociología Contemporánea. 2019. 6-19: 20-30.

\footnotetext{
* Correspondencia al Autor (Correo Electrónico: gpemorales74@ hotmail.com)

$\dagger$ Investigador contribuyendo como primer autor.
} 


\section{Introducción}

Los Ch'oles habitan en el sur de México en los estados de Campeche, Chiapas y Tabasco. En el estado de Tabasco se concentran en los municipios de Macuspana y Tacotalpa. Los Ch'oles de Tacotalpa se asentaron en un territorio habitado desde épocas prehispánicas por los Zoques (Terreros, 2006). Estos asentamientos fueron producto de la migración por conflictos armados y religiosos en comunidades de Chiapas.

Dichos procesos migratorios dieron como resultado la fundación de comunidades y el repoblamiento de las existentes. Los hombres y mujeres Ch'oles que migraron trajeron consigo su lengua, tradiciones, costumbres y una diversidad de especies animales y vegetales, dando como resultado la diversidad biocultural presente en este territorio.

En este sentido, a fin de delimitar y caracterizar la región que integra esta diversidad biocultural en el municipio de Tacotalpa es necesario establecer criterios que permitan establecerla o definirla como la región biocultural Ch'ol de Tacotalpa.

Se parte del planteamiento de Giménez (1996) quien señala que las regiones no deben considerarse como un dato a priori, sino un constructo fundado a partir de la interacción de diversos criterios -entre otros, los histórico culturales- con ciertos sistemas cuyas partes actúan en mayor medida entre sí que en relación con sistemas externos.

A pesar de la complejidad para establecer límites de lo que constituyen las regiones, la CDI (2006) estableció 25 regiones indígenas de México. Para tal efecto, se consideraron los municipios con más del $40 \%$ de población indígena, aspectos de carácter histórico, económico y lingüístico, contigüidad geográfica e inclusión de municipios con población indígena dispersa.

Considerando el ejercicio de regionalización anterior, para Tabasco se forma la región Chontal de Tabasco que comprende cinco municipios con población indígena entre los que se encuentra Tacotalpa a pesar de que predomina la lengua $\mathrm{Ch}$ 'ol.
Tal como lo señala Boege (2008), esta regionalización es útil para la implementación de las políticas públicas, sin embargo, las unidades territoriales del municipio y los límites de los estados poco coinciden con las de los pueblos indígenas.

En este sentido, la metodología propuesta por Boege (2008) para delimitar los territorios de los pueblos indígenas contemporáneos considerando los criterios de: localidades de $40 \%$ y más de hogares indígenas, contigüidad, ocupación espacial de las localidades en núcleos agrarios, límites municipales compartidos y contorno compartido por ejidos, comunidades o agrupaciones de pequeñas propiedades que tienen menos de $40 \%$ de hogares de población indígena permite establecer una superficie de 28 033092 hectáreas ( $14.3 \%$ del territorio nacional) en el que viven 8391944 habitantes, de los cuales $80.9 \%$ son población indígena.

Sin duda, en estas regiones se concentra una amplia biodiversidad que es aprovechada por las comunidades indígenas que han ocupado estos territorios. En este sentido, Toledo y OrtizEspejel, (2014) señalan que para la utilización de esta diversidad biológica se emplearon prácticas, saberes y conocimientos. Este saber-hacer sobre la naturaleza, el cual ha sido perfeccionado durante 9 mil años, es parte sustancial del Patrimonio Biocultural de México.

Considerando lo anterior, este trabajo tiene como propósito delimitar y caracterizar la región biocultural de Tacotalpa, Tabasco e identificar el patrimonio biocultural de las comunidades que la integran.

\section{Métodos}

Para delimitar la región se consideraron los criterios de localidades con $40 \%$ o más de población indígena y los ejidos ocupados por dichas localidades. La información sobre localidades con población indígena se obtuvo de la base de datos de la CDI (2010) y la información de los núcleos ejidales de la base de datos del RAN (2010). El mapa se elaboró con el software QGIS ver. 3.8. 
La caracterización de la región se realizó mediante investigación documental y de campo. En el primer caso, se buscó información documental sobre las comunidades y ejidos que comprenden la región delimitada, en el caso de investigación de campo se realizaron entrevistas y observación en seis comunidades representativas de la región.

La identificación del patrimonio biocultural se llevó a cabo mediante entrevistas semiestructuradas a sabios locales de seis localidades representativas y se contrastó con información documental reportada sobre el tema.

\section{Resultados y discusión}

Delimitación de región biocultural Ch’ol

Los hablantes de la lengua Ch'ol se distribuyen en los estados de Chiapas, Tabasco y Campeche. En el caso de Tabasco se localizan principalmente en los municipios de Macuspana, Tacotalpa y Tenosique (Figura 1).

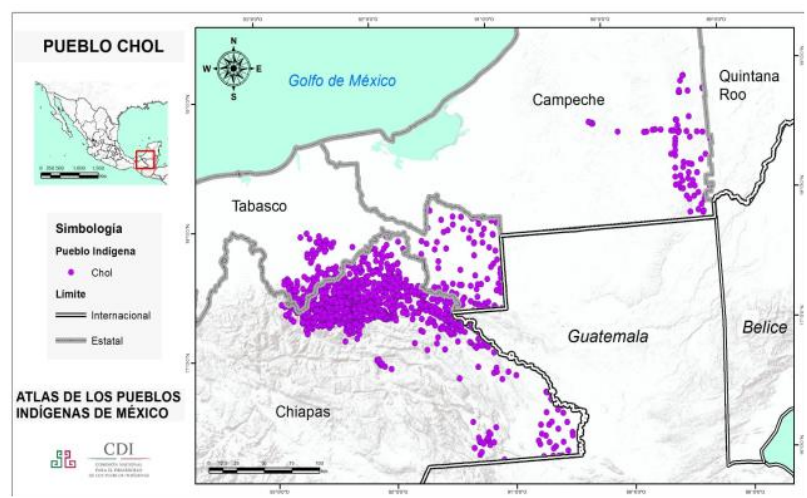

Figura 1 Territorio del pueblo ch'ol. Tomado de CDI 2015. Atlas de los pueblos indígenas de México

En Tabasco, gran parte de la población hablante de esta lengua se concentra en el municipio de Tacotalpa y actualmente ocupan el territorio históricamente habitado por los zoques. Como se aprecia en la figura 1, es difícil establecer los límites del territorio de los pueblos Ch'oles ya que en muchos casos las poblaciones se encuentran dispersas, lo que dificulta establecer la región de los Ch’oles de México.
La Comisión Nacional de Desarrollo de los Pueblos Indígenas (CDI) ha determinado "las regiones indígenas de México", sin embargo, señala que la definición de los límites de estas regiones no es tarea fácil debido a la compleja e intensa dinámica que caracteriza a los propios pueblos indígenas, la diversidad de grupos etnolingüísticos y sus variantes lingüísticas, sus volúmenes de población, patrones de asentamiento, modos de vida y formas de relación con el resto de la sociedad nacional (CDI, 2006).

Considerando elementos de las propuestas de CDI (2006) y Boege (2008) se propone una aproximación al territorio de los Ch'oles de Tacotalpa, Tabasco, denominada "Región Biocultural de los Ch'oles de Tacotalpa. Esta región está integrada por 31 localidades con más del $40 \%$ de población indígena, en su mayoría con alto grado de marginación y ocupan un territorio en 22 ejidos con una superficie de 22 414. 791 hectáreas (figura 2).

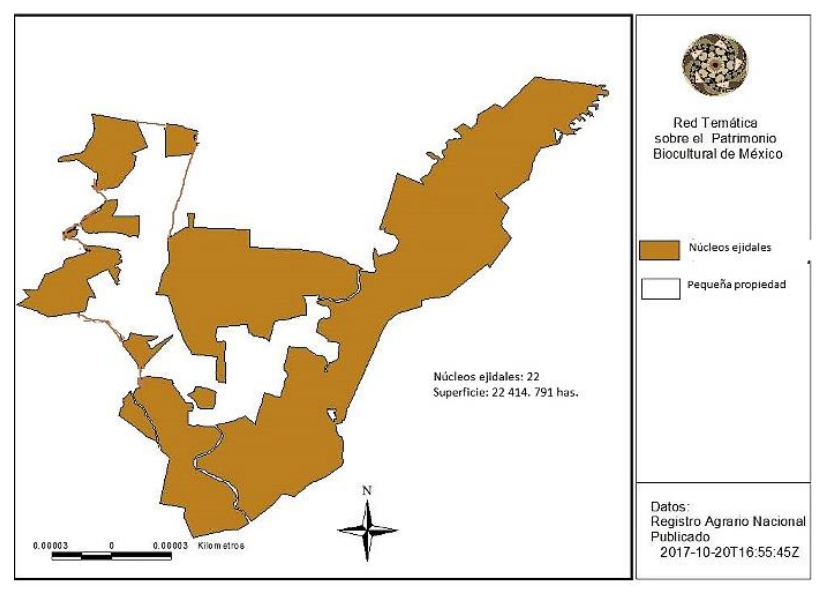

Figura 2 Región Biocultural Ch'ol de Tacotalpa, Tabasco Fuente: Carlos Martín Jiménez Arano con información del Registro Agrario Nacional

\section{Lengua y cultura Ch'ol.}

Como se mencionó anteriormente, la población Ch'ol de Tacotalpa es producto del desplazamiento de indígenas de Chiapas por conflictos religiosos, políticos y agrarios. En este sentido, los Ch'oles se concentra en comunidades colindantes con dicho estado. El $79.43 \%$ de los habitantes de las localidades que integran la región biocultural ch'ol de Tacotalpa hablan esta lengua. Las comunidades con la mayor cantidad de hablantes de la lengua Ch'ol son: Guayal, Carlos A. Madrazo, San Manuel, Agua Blanca, Libertad, Agua Escondida, Cuviac, entre otras (Tabla 1). 


\begin{tabular}{|c|c|c|c|c|}
\hline Localidad & $\begin{array}{c}\text { Grado de } \\
\text { Marginación }\end{array}$ & $\begin{array}{l}\text { Pob. } \\
\text { Total }\end{array}$ & $\begin{array}{l}\text { Pob. } \\
\text { Ind }\end{array}$ & $\begin{array}{c}\% \text { Pob } \\
\text { Ind. (\%) }\end{array}$ \\
\hline Agua Blanca & Alto & 592 & 592 & 100.0 \\
\hline $\begin{array}{l}\text { Carlos A. } \\
\text { Madrazo }\end{array}$ & Alto & 226 & 226 & 100.0 \\
\hline Cuviac & Alto & 376 & 376 & 100.0 \\
\hline Guayal & Alto & 954 & 947 & 99.3 \\
\hline Libertad & Alto & 1,042 & 1,028 & 98.7 \\
\hline San Manuel & Alto & 130 & 127 & 97.7 \\
\hline Noypac & Alto & 286 & 278 & 97.2 \\
\hline Puxcatán & Alto & 1,288 & 1,225 & 95.1 \\
\hline Buenos Aires & Alto & 253 & 236 & 93.3 \\
\hline $\begin{array}{l}\text { Cuauhtémoc } \\
\text { Barreal }\end{array}$ & Alto & 917 & 842 & 91.8 \\
\hline
\end{tabular}

Tabla 1 Localidades de Tacotalpa con mayor porcentaje de hablantes de lengua indígena

El Ch'ol es una lengua mayense, aunque diversos autores señalan que las variantes dialectales más importantes son la que se habla en Tila y Sabanilla y la que se habla en Tumbalá y Salto de Agua (Schumann, 1973; Pérez, 1993; Imberton, 2002) Por su parte, el Catálogo de lenguas indígenas nacionales señala que se autodenomina lakty'añ con dos variantes lingüísticas: la del sureste y la del noroeste. En las comunidades Ch'oles de Tacotalpa se habla la variante del noroeste.

Con la migración hacia el estado de Tabasco los Ch'oles llevaron y reprodujeron, además de su lengua, sus prácticas culturales, de esta forma, las comunidades Ch'oles mantienen los rasgos del pueblo Ch'ol como su cosmovisión, vida social, costumbres, fiestas y tradiciones.

En lo que respecta a su cosmovisión, Alejos y Martínez (2007) señalan que la concepción del mundo indígena maya ve al ser humano como parte de la naturaleza, es decir, lo sitúa dentro del entorno natural. En este sentido, los mayas construyen su identidad en íntima relación con un complejo de alteridades naturales, sociales y culturales que los circunda, lo cual los hace ser "legítimos", "originarios", es decir, hijos de la tierra, como brotados de ella, como "propios del lugar", al igual que las plantas y los animales. Por lo que la tierra es vista como un ser vivo, una entidad compleja, una alteridad vivida como madre, como fuente del sustento vital, y políticamente como territorio. Esto permite entender su relación con un entorno que poblaron recientemente. La organización tradicional de los Ch'oles se basa en la junta comunitaria, aunque en algunas localidades ha perdido fuerza; no obstante, se mantienen las mayordomías para organizar la vida social y religiosa.
La mayordomía presente en algunas localidades de Tacotalpa está a cargo de los ancianos varones, aunque se han dado casos de mujeres mayordomas y dado que entre los ch'oles predomina el catolicismo, su función principal es contribuir a la organización de las fiestas patronales. En este sentido, para los Ch'oles las fiestas patronales tienen mucha importancia por lo que a lo largo del año se celebran una serie de festividades en las diversas comunidades, entre las que destacan las siguientes:

\begin{tabular}{|l|l|l|}
\hline \multicolumn{1}{|c|}{ Fecha } & \multicolumn{1}{c|}{ Lugar } & $\begin{array}{l}\text { Fiesta } \\
\text { Fiesta del Señor de } \\
\text { Esquipulas. }\end{array}$ \\
\hline 2 de febrero & Cuitláhuac & $\begin{array}{l}\text { Fiesta de la Virgen } \\
\text { de la Candelaria }\end{array}$ \\
\hline 23 de mayo & Puxcatan & $\begin{array}{l}\text { Fiesta de la Virgen } \\
\text { María Auxiliadora }\end{array}$ \\
\hline 10 de Junio. & $\begin{array}{l}\text { Barreal } \\
\text { Cuauhtémoc }\end{array}$ & $\begin{array}{l}\text { Fiesta de Corpus } \\
\text { Christi o Señor de } \\
\text { Tila }\end{array}$ \\
\hline 24 de julio:: & Tapijulapa & $\begin{array}{l}\text { Fiesta de Santiago } \\
\text { Apóstol. }\end{array}$ \\
\hline 5 de agosto:: & Oxolotán & $\begin{array}{l}\text { Fiesta de Santo } \\
\text { Domingo de } \\
\text { Guzmán. de la }\end{array}$ \\
\hline $\begin{array}{l}\text { fuiesta de } \\
\text { diciembre }\end{array}$ & Guayal: & $\begin{array}{l}\text { Purísima } \\
\text { Concepción. }\end{array}$ \\
\hline
\end{tabular}

Tabla 2 Fiestas patronales en comunidades choles de Tacotalpa, Tabasco

Los Ch'oles son afectos a las danzas las cuales se representan sobre todo en carnaval (Fabregas-Puig, 2012).

Una de las más importantes es la danza del tigre del poblado Puxcatán, esta danza es de origen prehispánico y fue traída de Tila, Chiapas, forma parte del patrimonio biocultural de los Ch'oles por los elementos culturales y naturales que la integran (López et al., 2019). Otras tradiciones importantes so el Lavado de la ropa de los santos en Xicoténcatl, que, aunque es una tradición católica se practica por indígenas choles de esta comunidad; la bajada de santo sepulcro y la representación del viacrucis son otras tradiciones religiosas que se realizan en Oxolotán y finalmente la Pesca de la sardina, ritual de origen zoque pero practicado por los Ch'oles en Tapijulapa. Se identifican también ceremonias y rituales asociados a la actividad agrícola como la petición de agua de lluvia, la bendición de la tierra y la semilla (MariacaMendez et al., 2014). 
Sociodemografía de los Ch'oles.

En las 31 localidades que comprende la región biocultural Ch'ol habitan 13, 512 personas, de los cuales el $70.43 \%$ es población indígena (CDI, 2010). De la población de la región biocultural el $8 \%$ tiene más de 60 años, es la población que mantiene más las tradiciones y cultura, sin embargo, el $45 \%$ de la población está en el rango de los 6 a los 45 años de edad, en este periodo se considera que hay más peligro de que se pierda la lengua por la falta de la transmisión intergeneracional (UNESCO, 2003).

Con respecto a la utilización de la lengua, consideramos que algunas comunidades se pueden considerar como "paridad plurilingüe "; esto significa que en las comunidades existen dos lenguas, sin embargo, la que se utiliza en la mayoría de los ámbitos oficiales: el gobierno, la administración y la enseñanza, es la lengua castellana, mientras que la lengua originaria se utiliza en una serie de ámbitos públicos, especialmente en la interacción familiar y en aquellos lugares donde tienen trato social los miembros de las comunidades (UNESCO, 2003). y en otros ejidos, se considera que la región de ámbitos decrecientes en la lengua materna, esto significa que comienza a perder terreno con respecto a la lengua castellana y que solo se utiliza en pocos contornos familiares e incluso algunas miembros de la comunidad no la hablan. Por último, hay poblaciones en los cuales el porcentaje de población que habla la lengua materna representa el $40 \%$ de la población y es poco utilizada.

Con respecto a la educación en la región existen 25 escuelas de educación primaria, 10 secundarias, 3 bachilleratos y 1 institución de educación superior, sin embargo, el 9\% de la población no sabe leer ni escribir, hay 1819 (13\%) personas que tienen como máximo grado de estudio el sexto año de primaria, y existen 286 niños de 3 a 14 años de edad que no asisten a la escuela, esto contrasta con el $10 \%$ de la población que tiene estudios desde bachiller a maestría (INEGI, 2010).

Con respecto a la religión el 73\% pertenece a la religión católica y el $6 \%$ no está adscrito a ninguna religión, mientras que el resto de las religiones presentes tienen solo el $21 \%$. Para el 2010 solo menos del 3\% de la población tenía telefonía fija y de tipo celular.
Ambiente en la región biocultural Ch'ol.

Los Ch'oles tienen una muy importante relación directa con los elementos de la naturaleza que dieron origen a su pueblo y que les permiten mantener la vida en la tierra. Como menciona Boege (2008) los territorios y las regiones indígenas son centros intelectuales de origen y producción y diversificación biocultural donde se sobreponen la diversidad biológica y los ecosistemas que devienen en una línea de tiempo en paisajes (bio)culturales gestionados culturalmente. En este sentido, la situación ambiental en territorios Ch'oles de Tabasco está relacionada con los recursos naturales y la cultura, debido a procesos de desarrollo que han modificado en partes estos territorios de excepcional biodiversidad.

El municipio de Tacotalpa ocupa el $2.97 \%$ de la superficie del estado y cuenta con 91 localidades y una población total de 46, 302 habitantes. Presenta una población que se considera indígena con un 59.07\% $(25,301)$, sin embargo, la población de 3 años y más que habla alguna lengua indígena es de $26.46 \%(11,334)$ y de las personas que no hablan español fue el $2.30 \%$ (985.1) de los hablantes (INEGI, 2016)

El tipo de clima Af Cálido húmedo con lluvias todo el año (19.64\%) del territorio en Tabasco y que generalmente está distribuido en los municipios de Huimanguillo, Tacotalpa, Macuspana y Tenosique; prácticamente en la región montañosa. Las características específicas del clima $\mathrm{Af}(\mathrm{m}) \mathrm{w}$ "(i)g), presenta una temperatura media anual oscila entre 23 y $26^{\circ} \mathrm{C}$. El mes más caluroso es abril y el de menor temperatura es diciembre. El área es de las más lluviosas del país, registrando precipitaciones anuales entre 2900 y $3600 \mathrm{~mm}$. La temporada seca es de marzo a mayo; la mayor precipitación se presenta de agosto a octubre, según como reporta Arriaga, 2008.

En cuanto al clima que se presenta en comunidades situadas en cañadas, este grupo cultural ha desarrollado estrategias importantes para adaptarse a las condiciones; por ejemplo, casas elaboradas de caña con estructuras de madera permiten que durante las épocas de intenso calor puedan enfriarse rápidamente de noche. 
El tipo de clima también presenta en los últimos meses un incremento en la precipitación pluvial la cual tiene muchos efectos negativos en la agricultura y en los sistemas acuáticos como arroyos y ríos. La formación geológica de esta región se originó a partir de rocas calizas, quedando configurada con elevaciones pequeñas que alcanzan una altura de $1000 \mathrm{msnm}$. Los Ch'oles llaman a estas zonas con cubierta de vegetación arbórea "Montaña".

Las características que componen estos territorios se pueden encontrar pendientes hasta de un $38 \%$, donde principalmente se encuentran unidades geológicas calizas y lutitas del terciario superior. Zavala et al. (2016) mencionan que las características de estas zonas se les denomina paisajes de valles intermontanos, lomeríos y montañas, con alturas y pendientes de 40 a 1020 msnm y de 6 a $100 \%$. El valle es una porción del terreno alargada y plana, intercalada entre dos zonas circundantes de relieve alto generalmente drenadas por ríos. Los valles intermontanos $(0.4$ $\%)$ se localizan en Tacotalpa y Tenosique, tienen relieves de llanuras aluviales y deluviales; laderas sobre rocas detríticas y calizas, donde prevalecen procesos de erosión.

El lomerío es una porción del terreno quebrado, caracterizado por una repetición de colinas redondas o lomas alargadas, con cumbres a alturas variables, separadas por valles coluvio-aluviales. Los paisajes de lomerío suave a inclinado volcánico $(0.3 \%)$. y lomerío inclinado de areniscaslutitas-limonitas (2\%), se alternan con relieves convexos y valles erosivos. Las pendientes moderadas a fuertes, precipitaciones de 2500 a $4000 \mathrm{~mm}$ y la desforestación, contribuyen a los procesos de intemperización y erosión.

El paisaje de montaña se da como una porción del terreno, elevada, escabrosa, disectada y caracterizada por alturas importantes con relación a las unidades de paisajes circundantes de posición baja. Los suelos presentan una roca madre casi expuesta, con superficies orgánicas pobres y poco profundas. Presentan aluminio y fierro en exceso, en otras partes estos suelos son utilizados para agricultura de temporal. Para los choles existe una clasificación de suelo que tiene relación con los cultivos de laderas que pueda sostener alimentos para el autoconsumo.
Las unidades de suelo que se encuentran en la sierra son diversas, según INEGI pueden encontrarse Litosoles, Rendzina y Luvisoles (Larios y Hernández, S.F.). En esta parte serrana existen diversos programas de conservación de cuencas hidrográficas, pero sin resultado alguno debido a que estos suelos están expuestos a una fuerte erosión, provocando que en las partes bajas se depositen, por lo tanto, existe una red de drenaje superficial natural que origina en las planicies la deposición.

Estos suelos son clasificados por los choles considerando dos tipos categorías: uno incluye características que pueden ser percibidas por los sentidos como color, textura $y$ pedregosidad, y el otro tipo basado en su capacidad de uso agrícola y cobertura vegetal. Encontrándose, de acuerdo a la nomenclatura Ch'ol los siguientes suelos: Yiq'uel lum, Chachac lum/chıchıclumil, K'an kab lum, Ji'il lum or ji'lumil y Lum ambı ti xajlelol (SánchezHernández et al., 2018).

Además de clasificarlos, los Ch'oles en esta región tienen sus propias estrategias para la conservación de suelos, colocando muros de piedra y colocando los cultivos en formas de terrazas o colocación de arbustos en forma perpendicular a la pendiente.

El uso del suelo en la región Ch'ol está determinada principalmente por la relación directa de las prácticas culturales con el cultivo de maíz utilizando semillas criollas de las cuales se reportan 11 variedades (Morales y Padilla 2017); lo que define a los hombres del maíz, estas prácticas son de producción para el autoconsumo y de protección de la madre tierra.

El bosque tropical perennifolio fue el principal tipo de vegetación que tenía las áreas donde habitan los choles; sin embargo, debido a otras prácticas culturales se perdió gran parte del estado y actualmente existen reservas con situación de ejidos que pueden permanecer en su territorio. Existen especies que conforman este estrato de vegetación con alturas de $30 \mathrm{~m}$, representados por especies en peligro de extinción. 
Como mencionan Salazar et al. (2004), muchos de los campesinos utilizan ciertas especies que para ellos tienen más importancia por citar algunos como el palo mulato (Bursera simaruba), Bojón (Cordia alliodora), Caoba (Swietnia macrophyla), Cedro (Cedrela odorata), Guarumo (Cecropia obtusifolia), Guaya (Chamaedorea), Huapaque (Diallium guianense), Jobo (Spondias mombin) y Motusay (Phitodendrum radiatum).

También la siembra de maíz se considera prehispánico, agroecosistema donde muchos de los campesinos requieren de sus saberes con respecto al cosmo para su siembra; además de sembrar perpendicularmente a la pendiente para evitar erosión en los terrenos.

Se ha señalado el daño que muchos mamíferos silvestres hacen a diversos productos agrícolas (Hidalgo et al., 2016). Por ejemplo, En el ejido de Agua Blanca, el pecarí de collar y el venado temazate fueron identificados como los principales responsables del daño a los cultivos de frijol. En milpas de maíz del ejido de Oxolotán, del mismo municipio, el daño causado por mamíferos silvestres osciló entre el 10.8 y el $12.9 \%$ de la producción total.

Sin embargo, muchos campesinos han comentado (comunicación personal) que sus cultivos también dan para los animales, refiriéndose a que es parte de su responsabilidad, sembrar para que los animales silvestres también consuman lo que la tierra les da.

Según INEGI 1998, la superficie de uso común y distribución porcentual del uso actual reporta que Tacotalpa tiene un total de uso del suelo con 3, 054.079 ha, de las cuales el $62 \%$ corresponde a la parte agrícola, $78.9 \%$ forestal y otros con 14.9; esto indica que estas tierras son para conservar en ese territorio.

Sin embargo, la superficie parcelada tiene una distribución en el uso del suelo con 15, 163.046 ha, de las cuales el uso agrícola presenta 65.8\%, Ganadero 31.8\%, Agropecuario 0.4, forestal 0.5 y otros con 1.5; esto indica que las parcelas están destinadas mayormente a los cultivos y que se encuentran regularizadas y en gran parte han cambio sus prácticas hacia la ganadería.
A fines del siglo XVII y del siglo XVIII la población de la subregión Sierra aumentó al grado de concentrar la mayor densidad del estado (West et al. 1976, Tudela 1989). En este período se practicaba mucho la roza-tumbaquema, pero con aumento de explotación intensiva de maderas preciosas y de chicle (Tudela, 1989). Posteriormente con la entrada de la ganadería perjudico grandemente las áreas de selvas provocando la perdida de especies de árboles maderables, especie de mamíferos y muchas aves. Logrando así la reducción de áreas importantes para la conservación de la biodiversidad.

Los territorios Ch'oles son importantes porque tienen prácticas que permiten la sobrevivencia, como es la cacería de especies para su consumo o venta. Esto tiene relación con muchos conocimientos y saberes que tienen como parte de su cultura. Las especies de mamíferos que se prefieren a pesar de que están en peligro de extinción son por citar algunas: Venado, Puerco de monte, Armadillo y el tlacuache que es una especie perjudicial para estos grupos como señalan Hidalgo et al. (2016). Por otro lado, en sus casas tienen especies silvestres que forman parte de vivienda como son los mapaches, pericos, ardillas, monos, etc. Además, en algunos casos manejan las abejas de monte (Cano-Contreras et al., 2013).

En lo que respecta a la agrobiodiversidad, en los agroeosistemas locales se encuentra una amplia diversidad de especies. Para la milpa se reportan más de 50 especies entre las que destacan las variedades nativas de maíz. Dentro de estos agoecosistemas también resaltan las combinaciones entre diversas especies de chayas, frijol y calabaza. Las especies acuáticas existentes en los territorios Ch'oles tiene su forma muy particular asociado a las prácticas tradicionales relacionadas con arroyos que tienen aguas muy limpias donde se establecen especies que son consumidas por los pobladores, como en el caso de caracol (Pachychilus), cangrejo de río, camarón de río, bobo escama y sardinas. Sin embargo, las prácticas actuales con el uso de herbicidas y plaguicidas están aumentado el impacto negativo en diferentes estadios de vida de los invertebrados; por ejemplo, el Butox que se utiliza en los ríos para lograr una pesca abundante de camarón, sin importar otras especies acuáticas. 
La región hidrológica a la que pertenece el estado de Tabasco se denomina Grijalva Usumacinta, y la cuenca a la que ubica es R. Grijalva - Villahermosa. Derivadas de las subcuencas R. Tacotalpa (49.03\%), R. Puxcatán (24.03\%), R. Almandro (13.37\%), R. de la Sierra (9.88\%), R. Grijalva (2.28\%) y R. Macuspana $(1.41 \%)$, estas comprenden el territorio de los Ch'oles, donde existen corrientes de aguas perennes como Almandro, Amatán, Chinal, Chichilte, Cuncubac, El Zapotal, La Sierra, Lechugal, Nava, Puxcatán, Puyacatengo, San Nicolás y Tacotalpa; también existen otros intermitentes como: Murciélago, Azul, Tortuga y Seco (INEGI, 2016).

\section{Actividades productivas.}

Las actividades económicas giran en torno a la producción agropecuaria. Los agroecosistemas más importantes son la milpa, los huertos familiares y en menor medida los cacaotales y cafetales. La milpa es el sistema de producción más importante en el municipio de Tacotalpa, ya sea por la superficie de siembra o por la relevancia cultural de este sistema prehispánico. La milpa (con el maíz como cultivo más importante) se establece en dos ciclos, tornamil y milpa de año adaptada a las temporadas según las condiciones climáticas de la zona. La milpa incluye la producción de otras especies que sirven de complemento en la alimentación de las familias (calabaza, frijol, chile, chayas, tubérculos y otros) (figura 3) y de especies de mamíferos en peligro de extinción (Morales et al., 2015).
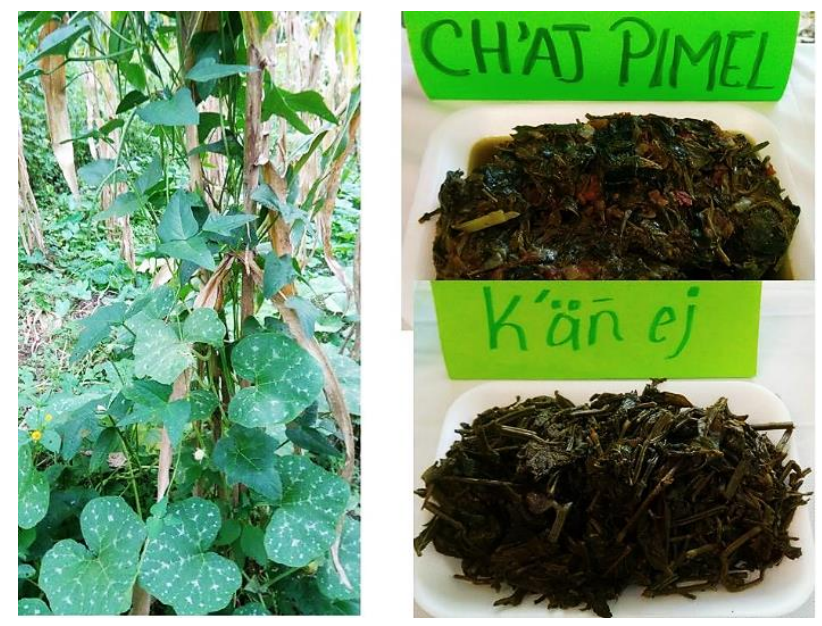

Figura 3 Especies y platillos obtenidos de la milpa en la región ch'ol de Tacotalpa, Tabasco
Para la región biocultural ch'ol de Tacotalpa, la milpa se realiza bajo condiciones de ladera para lo cual los campesinos han generado tecnología apropiada para garantizar el abasto de alimentos y reducir la pérdida de suelos (Morales et al., 2015). Sosa-Cabrera (2014) señala que la agricultura campesina Ch'ol, es un sistema adaptado al contexto ecológico y socioeconómico de la sierra de Tabasco; y ampliamente arraigado a las características culturales de los choles que habitan el municipio de Tacotalpa.

No obstante, es importante señalar el crecimiento de la ganadería y la superficie de palma de aceite y teca, aunque principalmente se ha dado en la planicie del municipio de Tacotalpa. Por otra parte, hay una tendencia a promover la actividad turística como apoyo económico a la región, aprovechando los recursos naturales y culturales (RodríguezOcaña, 2009) y el potencial de los agroecosistemas tradicionales (MoralesValenzuela, 2017).

\section{Patrimonio biocultural.}

Se identificaron algunos aspectos del patrimonio biocultural de la región, el cual está representado por la agricultura tradicional, la medicina tradicional, aprovechamiento de los recursos naturales, danzas y rituales. En el caso de la agricultura, predomina el cultivo de la milpa, que integra una amplia diversidad de especies con los que se preparan diversos platillos. Además de otros sistemas agroforestales ampliamente descritos por Moreno-Calles et al. (2013) como los huertos familiares y el cafetal. En lo que respecta a la medicina tradicional, los recursos terapéuticos incluyen a parteras, sobadores, curanderos, así como el uso de plantas, animales y hongos medicinales (García-Flores et al., 2019; Sosa et al., 2019; Villegas-Ramírez et al., 2019).

Los suelos, arroyos y ríos son aprovechados considerando las clasificaciones y uso de tecnologías locales. Se menciona el uso de hongos silvestres y otros recursos vegetales y animales como el ballil y el mimbre. Al respecto, Morales-Valenzuela et al. (2019) hacen una compilación de la memoria biocultural de los Ch'oles. 
Sin embargo, de acuerdo con Álvarez Gordillo et al. (2015) los habitantes de Tacotalpa reconocen que los patrimonios que dan sentido y continuidad a las comunidades son biológicos y culturales, identificando que las pérdidas durante los desastres han incrementado la vulnerabilidad social.

\section{Agradecimiento}

A la Red Temática sobre Patrimonio Biocultural del Consejo Nacional de Ciencia y Tecnología (CONACYT) por el apoyo para realización de este proyecto. Al Programa "Docentes en Vinculación" de la Universidad Intercultural del Estado de Tabasco por las facilidades otorgadas para el trabajo de campo. A los estudiantes de la Licenciatura en Desarrollo Rural Sustentable de la UIET por su apoyo en el trabajo de campo.

\section{Conclusiones}

La región biocultural Ch'ol de Tacotalpa, Tabasco está integrada por 31 localidades con más del $40 \%$ de población indígena, en su mayoría con alto grado de marginación, y ocupan un territorio de 22 414. 791 hectáreas.

En esta región se observan algunos relictos de selva mediana perennifolia en los que se usa y maneja una amplia biodiversidad.

El patrimonio biocultural de los Ch'oles de Tacotalpa, Tabasco está representado por la agricultura tradicional, la medicina tradicional, aprovechamiento de los recursos naturales y danzas y rituales.

\section{Referencias}

Alejos, J. \& Martínez, N. E. (2007). Ch'oles. Primera edición. México: Comisión Nacional para el Desarrollo de los Pueblos Indígenas.

Álvarez Gordillo, G. D. C., Vera Cortés, G., \& Ramos Muñoz, D. E. (2016). Vulnerabilidad y patrimonio biocultural en Tacotalpa, Tabasco. Política y cultura, (45), 211-239.

Arriaga, W. L. S. (2008). Avifauna en un Paisaje Antropizado en el Parque Estatal de la Sierra, Tabasco. Tesis de Doctor en Ciencias. El Colegio de la Frontera Sur.
Boege S. E. (2008). Las regiones bioculturales según los inventarios de la diversidad biológica domesticada y semidomesticada" en Boege S.E (coord.) El patrimonio biocultural de los pueblos indígenas de México. Hacia la conservación in situ de la biodiversidad y agrodiversidad en los territorios indígenas. México: INAH/ CDI, 158229.

Cano-Contreras, E. J., Martínez-Martínez, C., \& Balboa-Aguilar C. C. (2013). La "abeja de monte" (insecta:apidae, meliponini) de los choles de Tacotalpa, Tabasco: conocimiento local, presente y futuro. Etnobiología, 11(2), 4757.

Comisión Nacional para el Desarrollo De los pueblos indígenas (CDI). (2006). Regiones indígenas de México. México: CDI.

Comisión Nacional para el Desarrollo De los pueblos indígenas (CDI). (2010). Catálogo de localidades indígenas. México: CDI.

Fabregas-Puig, A. (2012) El mosaico chiapaneco: etnografía de las culturas indígenas. México: CDI.

García-Flores, J., González-Espinosa. M., Lindig-Cisneros, R., \& Casas, A. 2019. Traditional medicinal knowledge of tropical trees and its value for restoration of tropical forests. Botanical Sciences 97 (3), 336-354.

Giménez, Gilberto (1996). "Territorio y cultura". Estudios sobre las Culturas Contemporáneas, II (4), 9-30.

Hidalgo, M. M. G., Contreras, M. F. M., De la Cruz, A. J., Jiménez, D. D., Juárez, L. R., Oporto, P. S. \& Ávila, F. R. (2016). Mamíferos del estado de Tabasco. Pp. 441-472 en: BrionesSalas, M., Hortelano-Moncada, Y., MagañaCota, G., Sánchez-Rojas G. \& Sosa-Escalante, J. E. (eds.). Riqueza y Conservación de los Mamíferos en México a Nivel Estatal. México: Instituto de Biología, Universidad Nacional Autónoma de México, Asociación Mexicana de Mastozoología A. C. y Universidad de Guanajuato.

Imberton, G.M. (2002). La vergüenza: enfermedad y conflicto en una comunidad chol. México: UNAM, Programa de Investigaciones Multidisciplinarias sobre Mesoamérica y el Sureste. 
Instituto Nacional de Estadística, Geografía e Informática (INEGI). 1998. Tabasco. Tabulados básicos ejidales por municipio. Programa de certificación de derechos ejidales y titulación de solares urbanos, PROCEDE 1992-1997.

Instituto Nacional de Estadística, Geografía e Informática (INEGI). (2007). Censo agropecuario 2007.

Instituto Nacional de Estadística, Geografía e Informática (INEGI). (2010). Censo de d población y vivienda 2010.

Instituto Nacional de Estadística, Geografía e Informática. (2016). Panorama sociodemográfico de Tabasco 2015. 2016.

Larios, R. J. \& Hernández J. s/f. Zonificación fisiográfica del estado de Tabasco. Revista de Geografía Agrícola. 1-32 p.

López, L.; Ramos, D. \& Huicochea, L. (2019). Patrimonialización de la Danza del Tigre en el poblado ch'ol de Puxcatán, México. Culturales, 7, 1-41.

Mariaca-Méndez, R., Cano-Contreras, J.E., Morales-Valenzuela, G. \& Hernández-Sánchez, M. (2014). La milpa en la región serrana Chiapas-Tabasco de Huitiupán-Tacotalpa. En: González-Espinosa, M., \& Brunel-Manse, M. C. (coords.) Montañas, pueblos y agua. El Colegio de la Frontera Sur y Juan Pablos Editor. México. 323-359.

Morales-Valenzuela, G., Mariaca-Méndez, R., \& Padilla-Vega, J. (2015). Tecnología campesina en la milpa de ladera en Tacotalpa, Tabasco. En: Moreno, U. V. y Gómez, V. E. M. (Coord). Universidad Veracruzana Intercultural. México. 33-55.

Morales-Valenzuela, G. (2017). El potencial turístico de Tacotalpa: sistemas agroecológicos. Diálogos 52, 14-19.

Morales, V. G. \& Padilla V. J. (2017). Variedades locales de maíz en comunidades CH’oles de Tacotalpa, Tabasco. Revista de Ciencias Ambientales y Recursos Naturales, 3(7), 49-56.
Morales-Valenzuela, G. (2019). Avicultura de traspatio en Tacotalpa, Tabasco. En: MoralesValenzuela, G., Padilla-Vega, J. \& VásquezDávila, M.A. 2019. Memoria biocultural de la selva. Universidad Intercultural del Estado de Tabasco y Red Temática Sobre el Patrimonio Biocultural de México -CONACYT. 69-77.

Morales-Valenzuela, G., Padilla-Vega, J. \& Vásquez-Dávila, M. A. (2019). Memoria biocultural de la selva. Universidad Intercultural del Estado de Tabasco y Red Temática Sobre el Patrimonio Biocultural de México-CONACYT.

Moreno-Calles, A. I., Toledo, V. M., \& Casas, A. (2013). Los sistemas agroforestales tradicionales de México: una aproximación biocultural. Botanical Sciences, 91(4), 375-398.

Pérez, J.L. (1993). Los Choles de Tila y Su Mundo. BPR Publishers.

Rodríguez-Ocaña, L., Muñoz-Zetina, D.A., y López-Hernández, E.S. (2009). Etnoecoturismo como potencial de desarrollo local: el caso de la Sierra de Tacotalpa. México: UJAT.

Salazar, C. E. del C., Zavala C. J., Castillo A. O. y Cámara A. R. (2014). Evaluación espacial y temporal de la vegetación de la Sierra Madrigal, Tabasco, México (1973-2003). Investigaciones Geográficas, Boletín del Instituto de Geografía, UNAM, 54, 7-23.

Sánchez-Hernández, R., Méndez-De la Cruz, L., Palma-López, D. J., y Bautista-Zuñiga, F. (2018). Ch'ol nomenclature for soil classification in the ejido Oxolotán, Tacotalpa, Tabasco, México. Journal of ethnobiology and ethnomedicine, 14(1), 38.

Schumann, O. (1973). La lengua chol, de Tila (Chiapas). Universidad Nacional Autónoma de México.

Sosa-Cabrera, E. (2014) Agricultura chol de Tacotalpa, Tabasco. Tesis de Maestría. El Colegio de la Frontera Sur. 
Sosa, M. E., Padilla-Vega, J., \& Carreño-Ruiz, S. D. (2019). Los hongos comestibles y medicinales de Pomoquita, Tacotalpa, Tabasco: aspectos bioculturales para su conservación y aprovechamiento sustentable. En: En: MoralesValenzuela, G., Padilla-Vega, J. \& VásquezDávila, M. A. Memoria biocultural de la selva. Universidad Intercultural del Estado de Tabasco y Red Temática Sobre el Patrimonio Biocultural de México-CONACYT. 97-124. 78-96.

Terreros, E. E. (2006). Arqueología zoque de la región serrana tabasqueña. Estudios Mesoamericanos, 7, pp 29-43

Thomas, N.D., "Los Zoques", en: Esponda V. M. (1993). La población indígena de Chiapas, Tuxtla Gutiérrez, Gobierno del estado de Chiapas, Consejo Estatal de Fomento a la Investigación y Difusión de la Cultura, DIFChiapas/Instituto Chiapaneco de Cultura, pp. 4990.

Toledo, V.M. \& Ortiz-Espejel, B. 2014. México, regiones que caminan hacia la sustentabilidad: Una geopolítica de las resistencias bioculturales. Universidad Iberoamericana Puebla. 146 p.

Tudela, F. (1989). La modernización forzada del trópico: el caso de Tabasco. Proyecto integrado del Golfo. El Colegio de México: Instituto Politécnico Nacional, Centro de Investigación y de Estudios Avanzados.

Villegas-Ramírez, M. I., Pérez-Pérez, E., NiñoHernández, A. J. Villega-Jiménez, W. D., \& Sánchez-Domínguez, H. (2009). Usos medicinales de la fauna en Oxolotán, Tacotalpa, Tabasco. En: Morales-Valenzuela, G., PadillaVega, J. \& Vásquez-Dávila, M. A. Memoria biocultural de la selva. Universidad Intercultural del Estado de Tabasco y Red Temática Sobre el Patrimonio Biocultural de México-CONACYT. 97-124.

West, R. C., Psuty N. P \& Thom B. G. (1976). Las tierras bajas de Tabasco en el sureste de México. Gobierno del Estado de Tabasco, México.
Zavala, C. J., Jiménez, R. R., Palma, L. D. J. Bautista, Z. F. \& Gavi, R. F. 2016. Paisajes geomorfológicos: Base para el levantamiento de suelos en Tabasco, México. Ecosistemas y Recursos Agropecuarios, 3(8), 161-171. 